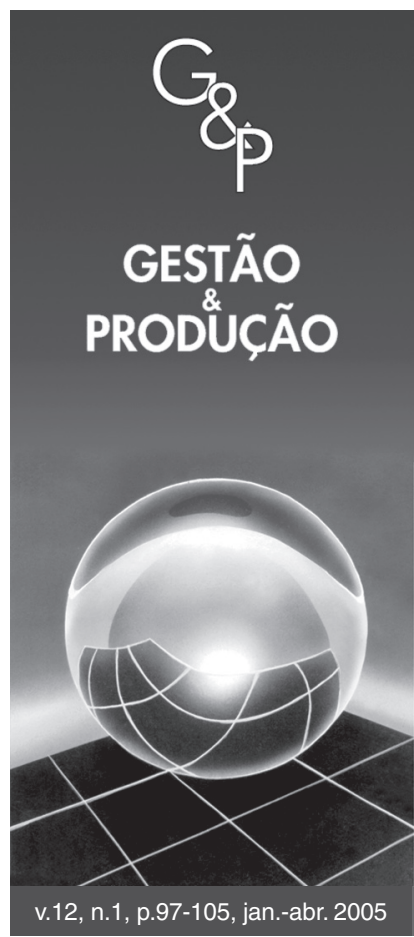

\title{
ESTIMADORES NÃO VICIADOS PARA O TEMPO MÉDIO ATÉ A FALHA E PARA PERCENTIS OBTIDOS DO MODELO DE REGRESSÃO DE WEIBULL
}

\author{
Linda Lee Ho \\ Departamento de Engenharia de Produção, \\ Escola Politécnica Universidade de São Paulo, EPUSP, \\ CEP 05508-900, São Paulo, SP, \\ e-mail: lindalee@usp.br
}

Aldy Fernandes da Silva

Centro Universitário Senac, Centro Universitário Álvares Penteado, Rua Engenheiro Eugênio Steveaux, 823, Prédio da Reitoria - Térreo, CEP 04696-000, São Paulo, SP , e-mail: aldy@ fecap.br

Recebido em 19/2/2004 Aceito em 15/2/2005

Resumo

Considere um modelo de confiabilidade descrito por uma regressão Weibull cujos parâmetros são estimadores pelo método da máxima verossimilhança. Eles serão utilizados para estimar outras quantidades como tempo médio de falha e quantis que por sua vez desempenham uma função importante numa análise de confiabilidade. O objetivo deste estudo é apresentar melhorias nos estimadores do tempo médio de falha e quantis uma vez que seus estimadores de máxima verossimilhança são viciados principalmente quanto envolve um tamanho de amostra reduzida. $O$ procedimento de reamostragem Bootstrap é proposto para corrigir estes vícios. Através de um estudo de simulação é possível quantificar o vício uma vez que sua determinação analítica é um tanto complicada. Um exemplo ilustra o procedimento proposto.

Palavras-chave: correção de vício, regressão Weibull, tempo médio até a falha, quantis, Bootstrap.

\section{Introdução}

Atualmente, os fabricantes enfrentam o desafio de desenvolver novos produtos com tecnologia mais avançada e em tempo recorde enquanto que, paralelamente, devem continuar os esforços de melhoria da produtividade, da confiabilidade e da qualidade de maneira geral. Melhorar a confiabilidade de um produto é sem dúvida um dos aspectos importantes de um programa de melhoria da qualidade. A viabilidade desse objetivo depende da realização de uma série de tarefas destinadas a avaliar diversos aspectos de seu desempenho, que constituem o que se costuma chamar de Análise de Confiabilidade (Nelson, 1982 e 1990). Em termos práticos, isto significa que as informações referentes à confiabilidade dos produtos necessitam ser obtidas em um período curto de tempo, para que possam ser utilizadas em novos projetos e na melhoria dos já existentes. Uma das metodologias utilizadas nessa etapa é a Análise do Tempo de Falha (testes realizados com o objetivo de verificar se o produto poderá proporcionar níveis de desempenho especificados durante sua vida operacional). Tal análise consiste em utilizar dados referentes à duração dos produtos (tempos até a falha) e modelá-los por meio de alguma distribuição de probabilidades (tais como Weibull, Log-Normal, Gama, etc.). Esta modelagem é realizada com dados experimentais referentes ao desempenho dos produtos e, geralmente, a Distribuição de Weibull é o modelo mais utilizado, uma vez que possui grande aplicabilidade na análise de dados derivados de experimentos industriais.

Em geral, a confiabilidade é definida como a probabilidade de um sistema ou item desempenhar satisfatoriamente a função requerida, sob condições de operação estabelecidas, por um período de tempo predeterminado (Lewis, 1996). Pela definição, confiabilidade depende diretamente do tempo, chamado tempo de vida (ou tempo até a falha). No entanto, esta definição não é fácil de ser assimilada, principalmente se for necessário comunicar-se 
com o público de modo geral. Para superar essa dificuldade, é comum que a confiabilidade seja também reportada de várias formas, utilizando-se uma ou mais das seguintes quantidades: tempo médio entre falhas de um item reparável (MTBF, mean time between failures); tempo médio até a falha de um item não reparável (MTTF, mean time to failure); o tempo no qual se espera que $p \times 100 \%$ dos produtos colocados em operação venham a falhar $\left(t_{p}\right.$, percentil de ordem $p$ ); e função de confiabilidade do item, que fornece a probabilidade do item funcionar por um período superior a $t(\mathrm{R}(t))$, entre outras.

Existem alguns trabalhos na literatura que desenvolveram correções em estimadores de máxima verossimilhança dos parâmetros da Distribuição de Weibull. Jacquelin (1993), Ross (1994), Ribeiro (1995) e Kahle (1996) são alguns deles. Entretanto, a maioria estudou apenas o vício dos estimadores dos parâmetros deste modelo no caso de dados não censurados e, não analisando, portanto, o comportamento desses vícios nas estimativas de quantidades como o tempo médio até a falha $(M T T F)$ e os percentis. Além disso, em experimentos industriais que geralmente são conduzidos com poucos dados amostrais, é comum a presença de censura e de fatores que permitem o controle do tempo até a falha do produto. Esses experimentos são modelados utilizando-se uma estrutura de regressão. Estudos desta natureza e baseados no modelo de regressão de Weibull não são encontrados na literatura.

O foco deste trabalho será um estudo do vício dos estimadores de máxima verossimilhança para o tempo médio até a falha e para o percentil de ordem $p$ do modelo de regressão de Weibull, quantidades estas que são usadas na análise de dados de durabilidade de experimentos industriais. Na Seção 2, a forma geral dos modelos de regressão de Weibull será apresentada, dando maior ênfase aos experimentos sob as mesmas condições (testes de vida) e à estimação de máxima verossimilhança das quantidades MTTF e percentis. Na Seção 3, a metodologia de reamostragem Bootstrap (Efron, 1979; Efron e Tibshirani, 1993) para redução do vício em E.M.V.s será descrita. Finalmente, resultados de estudos de simulação, visando avaliar o comportamento e a magnitude dos vícios dos E.M.V.s do MTTF e dos percentis de ordem 5\% e $10 \%$ obtidos do ajuste de modelos de regressão de Weibull, são apresentados na Seção 4. Esses resultados foram obtidos por meio da realização de experimentos de testes de vida para dados não censurados e com a presença de censura dos tipos I e II. Na Seção 5 um estudo de caso ilustra a aplicação da metodologia de correção de vício por reamostragem Bootstrap e, finalmente, as conclusões são apresentadas na Seção 6.

\section{Modelo de regressão de Weibull}

A família de distribuições Weibull é muito utilizada como um modelo para a distribuição dos tempos de falha na engenharia. O fato da sua taxa de falha ser monótona (ela é decrescente ou constante ou crescente conforme o parâmetro de forma) faz com que se torne bastante útil para modelar tempos de falha de equipamentos. Os modelos utilizados na análise de dados de confiabilidade são construídos para o logaritmo do tempo até a falha $T$, ou seja, $Y=\ln T$. Nestes modelos supõe-se que $Y$ tem uma distribuição com parâmetro de locação $\mu(\mathbf{x})$, em que $\mathbf{x}$ são variáveis regressoras e parâmetro de escala $\sigma>0$, que não depende das variáveis regressoras $\mathbf{x}$. Este será o procedimento geral, qualquer que seja a distribuição suposta para $Y$, neste caso Valor Extremo. Neste modelo, o parâmetro de locação da distribuição de $Y=\ln T$ é geralmente escrito em função de variáveis regressoras $\mathbf{x}$. $\mathrm{O}$ modelo toma então a seguinte forma geral

$$
Y=\ln T=\mu(\mathbf{x})+\sigma \varepsilon
$$

em que $\varepsilon$ tem distribuição do Valor Extremo que independe das variáveis regressoras $\mathbf{x}$ e $\mu(\mathbf{x})=\ln \alpha(\mathbf{x})=x \beta$ e $\sigma=1 / \delta$, onde $\alpha(\mathbf{x})$ e $\delta$ são, respectivamente, os parâmetros de escala e de forma de uma Distribuição de Weibull.

Notem que o modelo (1) toma a forma do modelo de regressão linear simples quando $Y=\ln T$ segue uma distribuição normal, com média (parâmetro de locação) $\mu(x)=x \beta$ e variância (parâmetro de escala) $\sigma^{2}$. Nos casos em que não há censura, este modelo pode ser ajustado com base na metodologia de Análise de Regressão (Neter et al., 1996). Entretanto, na presença de censura, como os casos que serão discutidos neste texto, essa metodologia não é adequada.

$\mathrm{Na}$ forma em que foi apresentado, o modelo de regressão em (1) é caracterizado por dois tipos de parâmetros: o parâmetro de forma $\delta$ e o de escala $\alpha(\mathrm{x})=\mathrm{e}^{\mathrm{x} \beta}$, em que $\boldsymbol{x}=(x 1 \ldots x \mathrm{p})$ e $\beta=(\beta 1 \ldots \beta 2)^{\prime}$. Essas quantidades conferem uma forma geral aos modelos probabilísticos. Entretanto, em cada estudo de confiabilidade, tais parâmetros devem ser estimados a partir das observações amostrais, de tal forma que o modelo fique determinado e possibilite estimar o MTTF e os Percentis de Ordem $p\left(t_{p}\right)$. O método de máxima verossimilhança talvez seja o método mais eficiente para esse tipo de dados. Ele incorpora as censuras, é relativamente simples de ser entendido e possui propriedades que permitem construir intervalos de confiança.

O modelo de regressão de Weibull mais simples encontrado na literatura é derivado de experimentos em que os produtos são submetidos às mesmas condições de testes. Estes experimentos são chamados de Testes de Vida e consideram que os dados são obtidos de forma que todas as fontes conhecidas de variabilidade ou fatores externos que possam afetar o tempo até a falha do produto são controladas. Supondo uma amostra aleatória de tempos até a falha de $n$ produtos, $t_{1}, t_{2}, \ldots, t_{n}$, o modelo de regressão, apresentado em (1), pode ser escrito como 


$$
y_{i}=\ln t_{i}=\mu+\sigma \varepsilon_{i}
$$

em que o tempo até a falha $t_{i}$ tem Distribuição de Weibull $(\alpha ; \delta)$ com função densidade de probabilidade

$$
f\left(t_{i}\right)=\frac{\delta}{\alpha^{\delta}} t_{i}^{s-1} \exp \left\{-\left(\frac{t_{i}}{\alpha}\right)^{\delta}\right\}, t_{i} \geq 0
$$

ou, de maneira equivalente, yi $=\ln$ ti tem distribuição do valor extremo $(\mu=\ln \alpha ; \sigma=1 / \delta)$, com a função de densidade de probabilidade dada por

$$
f(y)=\frac{1}{\sigma} \exp \left[\left(\frac{y-u}{\sigma}\right)-\exp \left(\frac{y-u}{\sigma}\right)\right],-\infty<y<+\infty
$$

Os parâmetros $(\alpha ; \delta)$ ou $(\mu ; \sigma)$ podem ser estimados pelo método da máxima verossimilhança. Usualmente, os estimadores de máxima verossimilhança (E.M.V.s) dos parâmetros deste modelo são viciados para pequenas amostras e tais vícios tendem a crescer à medida que o modelo incorpora algum mecanismo de censura. Conseqüentemente, os estimadores do MTTF e dos percentis são também viciados [maiores detalhes, ver Ferrari e Silva (1999) e Colosimo et al. (2000), uma vez que são funções destes parâmetros.

As quantidades de interesse MTTF e o Percentil de Ordem $p\left(t_{p}\right)$ são encontrados substituindo os estimadores de $\mu$ e $\sigma$ nas suas respectivas expressões, ou seja,

$$
\text { MTTF }=e \hat{\mu} \Gamma(1+\hat{\sigma})=\hat{\alpha} \Gamma\left(1+\frac{1}{\delta}\right)
$$

e,

$$
\hat{t}_{p}=\exp \{\hat{\mu}+\hat{\sigma} \ln [-\ln (1-p)]\}=\hat{\alpha}[-\ln (1-p)]^{1 / \hat{\delta}}
$$

As expressões das variâncias do $\hat{M T T F}$ e $\hat{t}_{p}$ são dadas, respectivamente, em (5) e (6):

$$
\begin{aligned}
& \operatorname{var}(\hat{M T T F}) \approx \operatorname{Var}(\hat{\alpha})\left(\frac{\partial M T T F}{\partial \alpha}\right)^{2} \\
& +2 \operatorname{Cov}(\hat{\alpha}, \hat{\delta}) \frac{\partial M T T F}{\partial \delta} \frac{\partial M T T F}{\partial \delta}+\operatorname{Var}(\hat{\delta})\left(\frac{\partial M T T F}{\partial \delta}\right)^{2} \\
& \mathrm{e}, \\
& \operatorname{Var}\left(\hat{t}_{p}\right) \approx \operatorname{Var}(\hat{\alpha})+[\ln (-\ln (1-p))]^{2} \\
& \operatorname{Var}(\hat{\delta})+2[\ln (-\ln (1-p))] \operatorname{Cov}(\hat{\alpha}, \hat{\delta}) \\
& \text { em que } \quad \frac{\partial M T T F}{\partial \alpha}=\Gamma(1+1 / \delta), \\
& \qquad \quad \frac{\partial M T T F}{\partial \delta}=-\left(\frac{\alpha}{\delta^{2}}\right) \Gamma(1+1 / \delta) \psi(1+1 / \delta) e \psi(z)
\end{aligned}
$$

é a função digamma definida como $\psi(\mathrm{z})=\operatorname{dlog} \Gamma(\mathrm{z}) /$ $\Gamma(\mathrm{z})$.

Estudos de simulação para este modelo foram realizados com o intuito de estudar o comportamento dos estimadores do MTTF e do Percentil de Ordem $p\left(t_{p}\right)$ com relação aos seus vícios.

\section{Redução de vício via Bootstrap}

A teoria de redução de vícios já não é tão recente na literatura estatística e teve seu desenvolvimento iniciado ou impulsionado pelo trabalho de Cox e Snell (1968). Desde então, vários trabalhos foram realizados para mostrar a importância desta metodologia dentro da teoria estatística e em outras áreas. É o que se pretende neste trabalho ao aplicar esta metodologia na Teoria da Confiabilidade usada em experimentos industriais.

Em um modelo regular (Cox e Hinkley, 1974) com um parâmetro unidimensional $\theta$, a expansão para o vício do E.M.V. $\hat{\theta}$ pode ser escrita como

$$
E(\hat{\theta}-\theta)=B(\theta)=\frac{B_{1}(\theta)}{n}+\frac{B_{2}(\theta)}{n^{2}}+\ldots
$$

em que $B_{1}(\theta)$ e $B_{2}(\theta)$ são funções de derivadas do logaritmo da verossimilhança com respeito ao parâmetro $\theta$ para uma observação simples e $n$ é usualmente o número de observações. A expansão em (7) também é válida no caso multidimensional e, nesta situação, uma expansão análoga a (7) é obtida para cada componente do vetor paramétrico. Usualmente, o vício do estimador $\theta$ é corrigido eliminando-se o termo $B_{1}(\theta)$ em (7). Uma forma de obter o termo $B_{1}(\theta)$ do vício é fazendo uso do processo de reamostragem intensivo denominado Bootstrap (Efron, 1979; Efron e Tibshirani, 1993).

A metodologia de reamostragem Bootstrap é uma técnica de grande relevância na literatura estatística e pode ser descrita da seguinte forma: Considere o princípio "plug-in", um método simples de estimação de parâmetros. O estimador "plug-in" de um parâmetro $\theta=\mathrm{g}(\mathrm{F})$ é definido por $\hat{\theta}=\mathrm{g}(\hat{F})$. Em outras palavras, a função $\theta=\mathrm{g}(F)$ da distribuição de probabilidade $F$ é estimada pela mesma função de distribuição empírica $\hat{F}, \hat{\theta}=$ $\mathrm{g}(\hat{F})$. O procedimento Bootstrap baseia-se simplesmente no princípio "plug-in". Suponha que se deseje estimar o vício do estimador de um parâmetro $\theta=\mathrm{g}(F)$ desconhecido. A distribuição $F$ é desconhecida, mas uma amostra aleatória $x=\left(x_{1}, \ldots, x_{n}\right)$ com função de distribuição empírica $\hat{F}$ pode ser obtida. Considere então um estimador $\hat{\theta}$ de $\theta$. O vício de $\hat{\theta}$ é definido como

$$
\text { vício }_{F}=\mathrm{E}_{F}(\hat{\theta})-\theta=\mathrm{E}_{F}(\hat{\theta})-\mathrm{g}(\mathrm{F})
$$

Retire uma amostra Bootstrap $x^{*}=\left(x^{*},{ }_{1}, \ldots, \mathrm{X}^{*}{ }_{n}\right)$ com distribuição de probabilidade empírica $\hat{F}$. Uma amostra Bootstrap $x^{*}, \ldots, x^{*}{ }_{n}$ é definida como uma amostra aleatória de tamanho $n$ retirada com reposição do conjunto de dados original $x_{1}, . ., x_{n}$. O Bootstrap assim definido é comumente chamado Bootstrap não-paramétrico e o estimador Bootstrap do vício de $\hat{\theta}$ é definido como

$$
\operatorname{vício}_{\hat{F}}=\mathrm{E}_{\hat{F}}\left(\hat{\theta}^{*}\right)-\mathrm{g}(\hat{\mathrm{F}})
$$

em que $\mathrm{E}_{\hat{F}}\left(\hat{\theta}^{*}\right)$ é a esperança de $\hat{\theta}$ sobre a distribuição de 
probabilidade empírica da amostra Bootstrap e $\mathrm{g}(\hat{F})$ é o estimador "plug-in" de $\theta$.

O estimador Bootstrap ideal do vício deve ser aproximado por um procedimento de simulação Monte Carlo. Selecione $B$ amostras Bootstrap independentes $x^{* 1}$, $x^{* 2}, \ldots, x^{* \mathrm{~B}}$ da distribuição empírica $F$. Calcule as réplicas Bootstrap $\hat{\theta}^{*}{ }_{\mathrm{b}}$ e aproxime a esperança $\mathrm{E}_{\hat{F}}\left(\hat{\theta}^{*}\right)$ pela média $\bar{\theta}_{B}^{*}=\sum_{b=1}^{B} \theta_{b}^{*} / B$. Este resultado é o estimador Bootstrap do parâmetro $\theta$. O estimador Bootstrap do erro padrão $\hat{\sigma}=\sigma(\hat{F})$ das $B$ réplicas Bootstrap $\hat{\theta}^{*}{ }_{\mathrm{b}}$, é dado por

$$
S_{B}^{*}=\sqrt{\frac{1}{B-1} \sum_{b=1}^{B}\left(\hat{\theta}_{b}^{*}-\bar{\theta}_{B}^{*}\right)^{2}}
$$

Finalmente, o estimador Bootstrap do vício de $\hat{\theta}$, de ordem $n^{-1}$, baseado nas $B$ réplicas é então dado por

$$
\text { vício }_{B}=\bar{\theta}_{B}^{*}-\hat{\theta}
$$

Como nosso objetivo é corrigir o vício do estimador $\hat{\theta}$ de um parâmetro $\theta$, então o estimador Bootstrap corrigido por vício (ECV) até ordem $n^{-1}$ é dado por

$$
\hat{\theta}_{B}=2 \hat{\theta}-\bar{\theta}_{B}^{*}
$$

A metodologia Bootstrap pode também ser utilizada quando os dados $\boldsymbol{x}=\left(x_{1}, \ldots, x_{n}\right)$ apresentam algum mecanismo de censura. Efron (1981) discutiu e apresentou o uso do Bootstrap para dados censurados à direita e para a construção de intervalos de confiança. Esta metodologia, assim como a apresentada por Efron (1981), foram aplicadas no desenvolvimento de estimadores corrigidos por vício para o tempo médio até a falha $(M T T F)$ e para o percentil de ordem $p\left(t_{p}\right)$ obtidos do modelo de Weibull para dados de testes de vida apresentado na Seção 2.

\section{Estudos de simulação}

Na Seção 3, o método de redução de vício por reamostragem Bootstrap foi apresentado como uma metodologia para auxiliar no desenvolvimento de correções por vício em estimadores de máxima verossimilhança. No entanto, o objetivo deste trabalho é o de obter correções para o vício dos E.M.V.s do tempo médio até a falha (MTTF) e dos percentis ( $5 \%$ e 10\%), quando estes são obtidos por meio do ajuste do modelo de Weibull a dados de tempos de falha.

$\mathrm{Na}$ metodologia de reamostragem Bootstrap procura-se reduzir o vício das estimativas de interesse por um processo computacional intensivo. De modo resumido, pode-se dizer que para uma dada amostra de tempos de falha, efetua-se, inicialmente, o cálculo dos estimadores de máxima verossimilhança dos parâmetros do modelo de Weibull e das respectivas quantidades MTTF e $t_{p}$ de interesse. O procedimento de reamostragem Bootstrap, então, consistiu de uma reamostragem sucessiva dos tempos de falha originais. No caso de reamostragem com dados censurados foi utilizada a metodologia descrita por Efron (1981). Cada reamostragem dos tempos de falha realizada foi denominada de réplica Bootstrap, sendo então calculada para esta, as E.M.V.s dos parâmetros do modelo de Weibull e as respectivas estimativas do MTTF e dos percentis de interesse. Após a realização de $\mathrm{B}=500$ réplicas Bootstrap, foi obtida a média das estimativas do MTTF e dos percentis e os seus respectivos vícios pelo uso da expressão (9). Finalmente, foi possível obter as estimativas corrigidas por vício (E.C.V.s) para as mesmas quantidades pela aplicação da expressão (10). Este tipo de procedimento denominado Bootstrap não-paramétrico (processo de reamostragem baseado na função de distribuição empírica dos tempos de falha) foi, portanto, utilizado nos estudos de simulação com experimentos de dados de testes de vida para a obtenção de correções no vício dos E.M.V.s das quantidades em estudo do modelo de Weibull. Os estudos de simulação apresentados neste trabalho foram desenvolvidos com o objetivo de avaliar a existência e a ordem de grandeza dos vícios dos estimadores de máxima verossimilhança (E.M.V.s) do MTTF e dos percentis, além de permitir a obtenção dos seus respectivos E.C.V.s por reamostragem Bootstrap. Esses estudos foram realizados utilizando um procedimento de Simulação Monte Carlo. Todas as simulações foram executadas por meio de programas desenvolvidos no "software" computacional Matlab 6.5 (2003), utilizando-se um total de 10.000 repetições do experimento.

Os resultados das simulações com dados censurados e não censurados obtidos para o MTTF e para os percentis $5 \%$ e 10\% estão apresentados nas Tabelas de 1 a 3. Nestas tabelas, estão disponibilizados os vícios relativos dos estimadores de máxima verossimilhança (E.M.V.s) e dos estimadores corrigidos por vício (E.C.V.s) quando comparados ao verdadeiro valor das quantidades de interesse (MTTF e percentis). Esses vícios foram obtidos de experimentos com dados não censurados e com mecanismos de censura do tipo I e do tipo II $(10 \%, 25 \%$ e $50 \%$ de censura nos dois tipos de censura), para os seguintes valores do parâmetro de forma $\delta$ iguais a 0,$75 ; 1,00$ e 1,50 do modelo de Weibull e para amostras de tamanhos iguais a $25 ; 50 ; 75 ; 100 ; 150 ;$ e 200.

De acordo com os resultados apresentados nas Tabelas de 1 a 3, pode-se concluir que as E.M.V.s geralmente apresentaram vícios consideráveis, principalmente, para os percentis $5 \%$ e $10 \%$. Além disso, as estimativas foram fortemente influenciadas pelo valor do parâmetro de forma $\delta$ do modelo de Weibull e pelo tamanho da amostra. $\mathrm{O}$ vício também aumenta quando aumenta a taxa de censura, tanto a do tipo I como a do tipo II.

De uma maneira geral, pode-se observar pelos resultados que os E.C.V.s apresentaram uma superioridade aos E.M.V.s no que diz respeito ao vício. Para experimentos 
Tabela 1. Vício relativo do E.M.V e E.C.V. do MTTF (parâmetro de escala $\alpha=10,000$ ).

\begin{tabular}{|c|c|c|c|c|c|c|c|c|c|c|c|c|c|c|c|}
\hline \multirow{3}{*}{$\frac{\delta}{(M T T F)}$} & \multirow[b]{3}{*}{$\mathrm{n}$} & \multirow{2}{*}{\multicolumn{2}{|c|}{$\begin{array}{c}\text { Sem } \\
\text { Censura }\end{array}$}} & \multicolumn{6}{|c|}{$\begin{array}{c}\text { Censura } \\
\text { Tipo I }\end{array}$} & \multicolumn{6}{|c|}{$\begin{array}{c}\text { Censura } \\
\text { Tipo II }\end{array}$} \\
\hline & & & & \multicolumn{2}{|c|}{$10 \%$} & \multicolumn{2}{|c|}{$25 \%$} & \multicolumn{2}{|c|}{$50 \%$} & \multicolumn{2}{|c|}{$10 \%$} & \multicolumn{2}{|c|}{$25 \%$} & \multicolumn{2}{|c|}{$50 \%$} \\
\hline & & EMV & $\mathrm{ECV}$ & EMV & $\mathrm{ECV}$ & EMV & ECV & EMV & ECV & EMV & ECV & EMV & $\mathrm{ECV}$ & EMV & ECV \\
\hline \multirow{6}{*}{$\begin{array}{l}0,75 \\
(11906,4)\end{array}$} & 25 & 1,01 & 1,00 & 1,03 & 0,98 & 1,07 & 0,83 & 1,29 & $*$ & 1,00 & 0,96 & 1,02 & 0,85 & 1,12 & $*$ \\
\hline & 50 & 1,00 & 1,00 & 1,01 & 0,99 & 1,04 & 0,99 & 1,14 & 0,64 & 1,00 & 0,98 & 1,00 & 0,96 & 1,01 & 0,85 \\
\hline & 75 & 1,00 & 1,00 & 1,01 & 1,00 & 1,02 & 0,99 & 1,07 & 0,96 & 1,00 & 0,98 & 1,00 & 0,97 & 1,00 & 0,91 \\
\hline & 100 & 1,00 & 1,00 & 1,00 & 1,00 & 1,01 & 1,00 & 1,06 & 0,98 & 1,00 & 0,99 & 1,00 & 0,98 & 1,00 & 0,94 \\
\hline & 150 & 1,00 & 1,00 & 1,01 & 1,00 & 1,01 & 1,00 & 1,03 & 0,99 & 1,00 & 0,99 & 1,00 & 0,99 & 1,00 & 0,96 \\
\hline & 200 & 1,00 & 1,00 & 1,00 & 1,00 & 1,01 & 1,00 & 1,02 & 1,00 & 1,00 & 0,99 & 1,00 & 0,99 & 1,01 & 0,98 \\
\hline \multirow{6}{*}{$\begin{array}{l}1,00 \\
(10000,0)\end{array}$} & 25 & 1,01 & 1,01 & 1,01 & 1,00 & 1,04 & 0,96 & 1,15 & $*$ & 1,00 & 0,98 & 1,00 & 0,95 & 1,05 & $*$ \\
\hline & 50 & 1,00 & 1,00 & 1,01 & 1,00 & 1,02 & 1,00 & 1,06 & 0,96 & 1,00 & 0,99 & 0,99 & 0,97 & 0,99 & 0,93 \\
\hline & 75 & 1,00 & 1,00 & 1,00 & 1,00 & 1,01 & 1,00 & 1,03 & 0,99 & 1,00 & 0,99 & 1,00 & 0,98 & 0,99 & 0,95 \\
\hline & 100 & 1,00 & 1,00 & 1,00 & 1,00 & 1,01 & 1,00 & 1,03 & 0,99 & 1,00 & 1,00 & 1,00 & 0,98 & 1,00 & 0,97 \\
\hline & 150 & 1,00 & 1,00 & 1,00 & 1,00 & 1,01 & 1,00 & 1,01 & 1,00 & 1,00 & 1,00 & 1,00 & 0,99 & 1,00 & 0,98 \\
\hline & 200 & 1,00 & 1,00 & 1,00 & 1,00 & 1,00 & 1,00 & 1,01 & 1,00 & 1,00 & 1,00 & 1,00 & 0,99 & 1,00 & 0,98 \\
\hline \multirow{6}{*}{$\begin{array}{l}1,50 \\
(9027,5)\end{array}$} & 25 & 1,00 & 1,00 & 1,00 & 1,00 & 1,01 & 0,99 & 1,05 & $*$ & 1,00 & 0,99 & 1,00 & 0,98 & 1,02 & 0,61 \\
\hline & 50 & 1,00 & 1,00 & 1,00 & 1,00 & 1,00 & 1,00 & 1,02 & 0,99 & 1,00 & 1,00 & 0,99 & 0,98 & 0,99 & 0,96 \\
\hline & 75 & 1,00 & 1,00 & 1,00 & 1,00 & 1,00 & 1,00 & 1,02 & 1,00 & 1,00 & 1,00 & 1,00 & 0,99 & 0,99 & 0,97 \\
\hline & 100 & 1,00 & 1,00 & 1,00 & 1,00 & 1,00 & 1,00 & 1,01 & 1,00 & 1,00 & 1,00 & 1,00 & 0,99 & 0,99 & 0,98 \\
\hline & 150 & 1,00 & 1,00 & 1,00 & 1,00 & 1,00 & 1,00 & 1,01 & 1,00 & 1,00 & 1,00 & 1,00 & 0,99 & 1,00 & 0,99 \\
\hline & 200 & 1,00 & 1,00 & 1,00 & 1,00 & 1,00 & 1,00 & 1,00 & 1,00 & 1,00 & 1,00 & 1,00 & 1,00 & 1,00 & 0,99 \\
\hline
\end{tabular}

* indica que a estimativa corrigida por vício (E.C.V.) obtida foi negativa.

Tabela 2. Vício relativo do E.M.V e E.C.V. do percentil 5\% (parâmetro de escala $\alpha=10,000$ ).

\begin{tabular}{|c|c|c|c|c|c|c|c|c|c|c|c|c|c|c|c|}
\hline \multirow[b]{2}{*}{$\delta$} & \multirow[b]{3}{*}{$\mathrm{n}$} & \multirow{2}{*}{\multicolumn{2}{|c|}{$\begin{array}{c}\text { Sem } \\
\text { Censura }\end{array}$}} & \multicolumn{6}{|c|}{$\begin{array}{c}\text { Censura } \\
\text { Tipo I }\end{array}$} & \multicolumn{6}{|c|}{$\begin{array}{c}\text { Censura } \\
\text { Tipo II }\end{array}$} \\
\hline & & & & \multicolumn{2}{|c|}{$10 \%$} & \multicolumn{2}{|c|}{$25 \%$} & \multicolumn{2}{|c|}{$50 \%$} & \multicolumn{2}{|c|}{$10 \%$} & \multicolumn{2}{|c|}{$25 \%$} & \multicolumn{2}{|c|}{$50 \%$} \\
\hline$\left(t_{0,5}\right)$ & & EMV & ECV & EMV & ECV & EMV & ECV & EMV & $\mathrm{ECV}$ & EMV & ECV & EMV & ECV & EMV & $\mathrm{ECV}$ \\
\hline \multirow{6}{*}{$\begin{array}{l}0,75 \\
(190,6)\end{array}$} & 25 & 1,44 & 0,95 & 1,46 & 0,91 & 1,50 & 0,94 & 1,57 & $*$ & 1,53 & 1,05 & 1,60 & 1,06 & 1,73 & 1,09 \\
\hline & 50 & 1,20 & 0,99 & 1,21 & 0,98 & 1,22 & 0,98 & 1,25 & 0,97 & 1,23 & 1,02 & 1,28 & 1,05 & 1,32 & 1,05 \\
\hline & 75 & 1,13 & 0,99 & 1,13 & 0,99 & 1,14 & 0,99 & 1,17 & 0,99 & 1,15 & 1,01 & 1,18 & 1,04 & 1,22 & 1,05 \\
\hline & 100 & 1,10 & 0,99 & 1,10 & 0,99 & 1,11 & 1,00 & 1,12 & 0,99 & 1,11 & 1,01 & 1,13 & 1,03 & 1,16 & 1,03 \\
\hline & 150 & 1,07 & 1,00 & 1,07 & 1,00 & 1,08 & 1,01 & 1,08 & 1,00 & 1,07 & 1,01 & 1,09 & 1,02 & 1,11 & 1,02 \\
\hline & 200 & 1,05 & 1,00 & 1,04 & 0,99 & 1,05 & 1,00 & 1,06 & 1,00 & 1,05 & 1,01 & 1,06 & 1,01 & 1,08 & 1,02 \\
\hline \multirow{6}{*}{$\begin{array}{l}1,00 \\
(512,9)\end{array}$} & 25 & 1,26 & 1,00 & 1,27 & 0,99 & 1,27 & 0,98 & 1,31 & $*$ & 1,29 & 1,05 & 1,33 & 1,07 & 1,39 & 1,08 \\
\hline & 50 & 1,12 & 1,00 & 1,13 & 1,00 & 1,12 & 0,99 & 1,15 & 1,00 & 1,14 & 1,03 & 1,17 & 1,04 & 1,20 & 1,06 \\
\hline & 75 & 1,08 & 1,00 & 1,09 & 1,00 & 1,09 & 1,00 & 1,10 & 1,00 & 1,09 & 1,02 & 1,10 & 1,02 & 1,13 & 1,03 \\
\hline & 100 & 1,06 & 1,00 & 1,06 & 1,00 & 1,06 & 1,00 & 1,07 & 1,00 & 1,07 & 1,01 & 1,07 & 1,02 & 1,10 & 1,03 \\
\hline & 150 & 1,04 & 1,00 & 1,04 & 1,00 & 1,04 & 1,00 & 1,04 & 1,00 & 1,04 & 1,00 & 1,05 & 1,01 & 1,06 & 1,02 \\
\hline & 200 & 1,03 & 1,00 & 1,03 & 1,00 & 1,03 & 1,00 & 1,03 & 1,00 & 1,04 & 1,01 & 1,04 & 1,01 & 1,05 & 1,01 \\
\hline \multirow{6}{*}{$\begin{array}{l}1,50 \\
(1380,5)\end{array}$} & 25 & 1,13 & 1,01 & 1,12 & 0,99 & 1,12 & 0,99 & 1,14 & 0,79 & 1,15 & 1,04 & 1,16 & 1,05 & 1,20 & 1,04 \\
\hline & 50 & 1,06 & 1,00 & 1,06 & 1,00 & 1,06 & 1,00 & 1,08 & 1,01 & 1,08 & 1,02 & 1,08 & 1,03 & 1,10 & 1,03 \\
\hline & 75 & 1,04 & 1,00 & 1,04 & 1,00 & 1,04 & 1,00 & 1,04 & 1,00 & 1,05 & 1,01 & 1,05 & 1,02 & 1,06 & 1,02 \\
\hline & 100 & 1,03 & 1,00 & 1,03 & 1,00 & 1,03 & 1,00 & 1,04 & 1,00 & 1,03 & 1,01 & 1,04 & 1,01 & 1,05 & 1,02 \\
\hline & 150 & 1,02 & 1,00 & 1,02 & 1,00 & 1,02 & 1,00 & 1,02 & 1,00 & 1,02 & 1,01 & 1,03 & 1,01 & 1,03 & 1,01 \\
\hline & 200 & 1,01 & 1,00 & 1,01 & 1,00 & 1,01 & 1,00 & 1,02 & 1,00 & 1,02 & 1,00 & 1,02 & 1,01 & 1,02 & 1,01 \\
\hline
\end{tabular}

* indica que a estimativa corrigida por vício (E.C.V.) obtida foi negativa.

em que realmente os E.M.V.s do MTTF e dos percentis foram viciados, o procedimento de reamostragem Bootstrap conseguiu reduzir satisfatoriamente o vício dessas estimativas, fazendo com que as E.C.V.s fossem bem próximas do verdadeiro valor. Essa redução só não foi satisfatória quando o experimento foi realizado com altas taxas de censura (50\%) e tamanho de amostras muito pequenas $(n=25)$, para valores de $\delta$ iguais a 0,75 e 1,00 . 
Tabela 3. Vício relativo do E.M.V e E.C.V. do percentil $10 \%$ (parâmetro de escala $\alpha=10,000$ ).

\begin{tabular}{|c|c|c|c|c|c|c|c|c|c|c|c|c|c|c|c|}
\hline \multirow[b]{2}{*}{$\delta$} & \multirow[b]{3}{*}{$\mathrm{n}$} & \multirow{2}{*}{\multicolumn{2}{|c|}{$\begin{array}{c}\text { Sem } \\
\text { Censura }\end{array}$}} & \multicolumn{6}{|c|}{$\begin{array}{c}\text { Censura } \\
\text { Tipo I }\end{array}$} & \multicolumn{6}{|c|}{$\begin{array}{c}\text { Censura } \\
\text { Tipo II }\end{array}$} \\
\hline & & & & \multicolumn{2}{|c|}{$10 \%$} & \multicolumn{2}{|c|}{$25 \%$} & \multicolumn{2}{|c|}{$50 \%$} & \multicolumn{2}{|c|}{$10 \%$} & \multicolumn{2}{|c|}{$25 \%$} & \multicolumn{2}{|c|}{$50 \%$} \\
\hline$\left(t_{0}\right)$ & & EMV & ECV & EMV & ECV & EMV & EC & EMI & ECV & EMV & $\mathrm{ECV}$ & EMI & ECV & EMV & $\mathrm{ECV}$ \\
\hline \multirow{6}{*}{$\begin{array}{l}0,75 \\
(497,6)\end{array}$} & 25 & 1,29 & 0,99 & 1,28 & 0,97 & 1,30 & 0,9 & 1,32 & $*$ & 1,33 & 1,05 & 1,35 & 1,06 & 1,42 & 1,08 \\
\hline & 50 & 1,13 & 1,00 & 1,13 & 1,00 & 1,14 & 0,9 & 1,14 & 0,99 & 1,15 & 1,02 & 1,17 & 1,04 & 1,18 & 1,03 \\
\hline & 75 & 1,09 & 1,00 & 1,08 & 1,00 & 1,09 & 1,0 & 1,10 & 1,00 & 1,09 & 1,01 & 1,11 & 1,03 & 1,13 & 1,03 \\
\hline & 100 & 1,06 & 1,00 & 1,06 & 1,00 & 1,07 & 1,0 & 1,07 & 0,99 & 1,07 & 1,01 & 1,08 & 1,02 & 1,09 & 1,02 \\
\hline & 150 & 1,05 & 1,00 & 1,04 & 1,00 & 1,05 & 1,0 & 1,05 & 1,00 & 1,05 & 1,01 & 1,06 & 1,01 & 1,06 & 1,01 \\
\hline & 200 & 1,03 & 1,00 & 1,03 & 0,99 & 1,03 & 1,0 & 1,04 & 1,00 & 1,03 & 1,00 & 1,04 & 1,01 & 1,05 & 1,01 \\
\hline \multirow{6}{*}{$\begin{array}{l}1,00 \\
(1053,6)\end{array}$} & 25 & 1,17 & 1,01 & 1,17 & 1,00 & 1,16 & 1,0 & 1,18 & * & 1,19 & 1,04 & 1,21 & 1,05 & 1,23 & 1,05 \\
\hline & 50 & 1,08 & 1,00 & 1,08 & 1,01 & 1,07 & 1,0 & 1,09 & 1,00 & 1,09 & 1,02 & 1,11 & 1,03 & 1,12 & 1,03 \\
\hline & 75 & 1,06 & 1,00 & 1,06 & 1,00 & 1,06 & 1,0 & 1,06 & 1,00 & 1,06 & 1,01 & 1,06 & 1,01 & 1,07 & 1,02 \\
\hline & 100 & 1,04 & 1,00 & 1,04 & 1,00 & 1,04 & 1,0 & 1,04 & 1,00 & 1,05 & 1,01 & 1,05 & 1,01 & 1,06 & 1,01 \\
\hline & 150 & 1,03 & 1,00 & 1,02 & 1,00 & 1,03 & 1,0 & 1,02 & 0,99 & 1,02 & 1,00 & 1,03 & 1,01 & 1,04 & 1,01 \\
\hline & 200 & 1,02 & 1,00 & 1,02 & 1,00 & 1,02 & 1,0 & 1,02 & 1,00 & 1,03 & 1,01 & 1,02 & 1,00 & 1,03 & 1,01 \\
\hline \multirow{6}{*}{$\begin{array}{l}1,50 \\
(2230,8)\end{array}$} & 25 & 1,09 & 1,01 & 1,08 & 1,00 & 1,08 & 1,0 & 1,09 & 0,79 & 1,10 & 1,03 & 1,10 & 1,03 & 1,13 & 1,02 \\
\hline & 50 & 1,04 & 1,00 & 1,04 & 1,00 & 1,04 & 1,0 & 1,05 & 1,00 & 1,05 & 1,02 & 1,05 & 1,02 & 1,06 & 1,02 \\
\hline & 75 & 1,03 & 1,00 & 1,03 & 1,00 & 1,02 & 1,0 & 1,03 & 1,00 & 1,03 & 1,01 & 1,04 & 1,01 & 1,04 & 1,01 \\
\hline & 100 & 1,02 & 1,00 & 1,02 & 1,00 & 1,02 & 1,0 & 1,02 & 1,00 & 1,02 & 1,01 & 1,03 & 1,01 & 1,03 & 1,01 \\
\hline & 150 & 1,01 & 1,00 & 1,01 & 1,00 & 1,01 & 1,0 & 1,01 & 1,00 & 1,02 & 1,00 & 1,02 & 1,01 & 1,02 & 1,01 \\
\hline & 200 & 1,01 & 1,00 & 1,01 & 1,00 & 1,01 & 1,0 & 1,01 & 1,00 & 1,01 & 1,00 & 1,01 & 1,00 & 1,01 & 1,00 \\
\hline
\end{tabular}

* indica que a estimativa corrigida por vício (E.C.V.) obtida foi negativa.

\section{Estudo de caso}

Este estudo de caso consistiu de uma análise do conjunto de dados sobre o tempo até a quebra de um tipo de isolante elétrico sujeito a um estresse de voltagem constante apresentado em Lawless (1982). Os dados apresentados na Tabela 4 são não censurados e representam os tempos (em minutos) até a quebra, para quatro grupos de isolantes submetidos a diferentes níveis de voltagem.

Lawless (1982) utilizou este conjunto de dados para ilustrar o ajuste do modelo de Weibull a dados de falha, tendo calculado, portanto, somente as estimativas dos seus parâmetros, ou seja, $\alpha$ e $\delta$. A análise realizada em Lawless (1982) consistiu do ajuste de um modelo de Weibull para os tempos até a quebra, a partir de um dado nível de voltagem fixo.

Neste trabalho, utilizamos os dados de tempos até a quebra, em cada um dos seus níveis, apenas para ilustrar uma situação de experimento de teste de vida sem a presença de covariável e com diversos tamanhos de amostra. Esse conjunto de dados serviu a esse propósito e, portanto, a análise dos dados da Tabela 4 foi realizada ajustando-se um modelo de Weibull para cada nível de voltagem, conforme realizado em Lawless (1982). O ajuste do modelo consistiu em calcular, para cada nível de voltagem, as estimativas de máxima verossimilhança (E.M.V.s) dos parâmetros do modelo de Weibull, do MTTF e dos percentis de ordem 5\% e 10\%. Além dessas, foram calculadas as estimativas corrigidas por vício (E.C.V.s) pelo procedimento de reamostragem Bootstrap para as quantidades MTTF e percentis. As estimativas corrigidas por vício foram encontradas utilizando-se um total de 5000 réplicas Bootstrap. Para cada modelo ajustado, foram calculadas também as estimativas por intervalo para as quantidades de interesse. Os intervalos para as E.M.V.s do MTTF foram obtidos fazendo-se o uso da expressão da variância estimada em (5), [maiores detalhes ver em Colosimo e Ho (1999)]. Já os intervalos de confiança para as E.M.V.s dos percentis foram obtidos fazendo-se o uso da expressão da variância dada em (6). Os intervalos para as E.C.V.s por reamostragem Bootstrap foram construídos pelo Método do Percentil Corrigido por Vício (Bias-Corrected Percentile Bootstrap - BCPB) segundo Efron e Tibshirani (1986). Os resultados das estimativas estão apresentados na Tabela 5.

Observando os resultados apresentados na Tabela 5, podemos concluir que não houve diferenças consideráveis entre as E.M.V.s e as E.C.V.s para o MTTF obtidas para os tempos de quebra dos isolantes. No entanto, com relação aos percentis, podemos observar grandes diferenças quando comparamos as estimativas sem correção $\left(t_{0,05 B}, t_{0,10 B}\right)$ com as estimativas corrigidas por vício $\left(t_{0,05 B}\right.$, $\left.t_{0,10 B}\right)$. Além disso, os intervalos obtidos para as estimativas corrigidas por reamostragem Bootstrap apresentaram menor amplitude quando comparados com os intervalos obtidos para as estimativas de máxima verossimilhança.

Voltando aos resultados dos estudos de simulação descritos nas Tabelas de 1 a 3 pode-se observar que, para os experimentos com dados não censurados, o E.M.V. do MTTF apresentou praticamente vício próximo de zero; 
isso ocorreu até para amostras de tamanho igual a 25 . No entanto, para os percentis, mesmo com dados não censurados e amostras iguais a 25 , os experimentos de simulação mostraram que os E.M.V.s são bastante viciados. Esses resultados, portanto, ajudam a explicar as grandes

Tabela 4. Tempos de falha de um tipo de isolante elétrico.

\begin{tabular}{ccrrr}
\hline $\begin{array}{c}\text { Número } \\
\text { da } \\
\text { unidade }\end{array}$ & \multicolumn{4}{c}{ Nível de Voltagem $(\mathbf{k V})$} \\
& $\mathbf{3 0}$ & $\mathbf{3 2}$ & $\mathbf{3 4}$ & $\mathbf{3 6}$ \\
& $\boldsymbol{n = 1 1}$ & $\boldsymbol{n = 1 5}$ & $\boldsymbol{n = 1 9}$ & $\boldsymbol{n}=\mathbf{1 5}$ \\
\hline 1 & 17,05 & 0,40 & 0,96 & 1,97 \\
2 & 22,66 & 82,85 & 4,15 & 0,59 \\
3 & 21,02 & 9,88 & 0,19 & 2,58 \\
4 & 175,88 & 89,29 & 0,78 & 1,69 \\
5 & 139,07 & 215,10 & 8,01 & 2,71 \\
6 & 144,12 & 2,75 & 31,75 & 25,50 \\
7 & 20,46 & 0,79 & 7,35 & 0,35 \\
8 & 43,40 & 15,93 & 6,50 & 0,99 \\
9 & 194,90 & 3,91 & 8,27 & 3,99 \\
10 & 47,30 & 0,27 & 33,91 & 3,67 \\
11 & 7,74 & 0,69 & 32,52 & 2,07 \\
12 & & 100,58 & 3,16 & 0,96 \\
13 & & 27,80 & 4,85 & 5,35 \\
14 & & 13,95 & 2,78 & 2,90 \\
15 & & 53,24 & 4,67 & 13,77 \\
16 & & & 1,31 & \\
17 & & & 12,06 & \\
18 & & & 36,71 & \\
19 & & & 72,89 & \\
\hline
\end{tabular}

diferenças encontradas entre as estimativas de máxima verossimilhança e as estimativas corrigidas por vício para os percentis $5 \%$ e $10 \%$ observadas na Tabela 5 .

\section{Conclusões}

Estruturas importantes em estudos de durabilidade, tais como o tempo médio até a falha e o percentil de ordem p, têm grande uso na indústria. O desenvolvimento de estimadores não viciados para essas quantidades pode trazer ganhos substanciais no estudo da confiabilidade de produtos. Estes ganhos podem ser fundamentais no desenvolvimento de novos produtos, uma vez que podem diminuir custos relativos aos processos de desenvolvimento e experimentação de produtos. Além disso, podem até mesmo permitir, quando possível, a redução do tamanho da amostra em planejamentos experimentais com dados de testes de vida. Essa redução do número de dados coletados proporcionará uma redução de custo e tempo em tais estudos.

Os resultados apresentados neste trabalho ilustram a importância de correções de vício em E.M.V.s. A ênfase deste trabalho foi no estudo do comportamento do vício de quantidades que são muito utilizadas em estudos de durabilidade de produtos, o MTTF e os percentis, e não nos parâmetros do modelo de Weibull, como é comum encontrar na literatura. A atenção foi centralizada na estimação dessas quantidades quando obtidas do ajuste do modelo de Weibull a dados de tempos de falha. Os resultados foram satisfatórios. As estimativas de máxima verossimilhança do MTTF e, principalmente, dos percentis $5 \%$ e $10 \%$ apresentaram, nos estudos de

Tabela 5. Estimativas do ajuste do modelo de Weibull para os tempos até a quebra de um tipo de isolante elétrico.

\begin{tabular}{|c|c|c|c|c|}
\hline \multicolumn{5}{|c|}{ Nível de Voltagem e Tamanho da Amostra } \\
\hline $\begin{array}{c}\text { Quantidades de } \\
\text { Interesse }\end{array}$ & $\begin{array}{c}30 \\
n=11\end{array}$ & $\begin{array}{c}32 \\
n=15\end{array}$ & $\begin{array}{c}34 \\
n=19\end{array}$ & $\begin{array}{c}36 \\
n=15\end{array}$ \\
\hline$\alpha$ & 77,58 & 25,94 & 12,22 & 4,29 \\
\hline$\delta$ & 1,06 & 0,56 & 0,77 & 0,89 \\
\hline $\begin{array}{l}\text { MTTF } \\
\text { I.C. }(95 \%)\end{array}$ & $\begin{array}{c}75,86 \\
(33,51 ; 118,21)\end{array}$ & $\begin{array}{c}42,81 \\
(2,12 ; 83,49)\end{array}$ & $\begin{array}{c}14,24 \\
(5,86 ; 22,61)\end{array}$ & $\begin{array}{c}4,55 \\
(1,96 ; 7,13)\end{array}$ \\
\hline $\begin{array}{l}\text { MTTF } \\
\text { I.C. }(95 \%)\end{array}$ & $\begin{array}{c}75,79 \\
(44,03 ; 111,98)\end{array}$ & $\begin{array}{c}42,82 \\
(21,01 ; 77,44)\end{array}$ & $\begin{array}{c}14,30 \\
(8,47 ; 22,82)\end{array}$ & $\begin{array}{c}4,55 \\
(2,39 ; 8,16)\end{array}$ \\
\hline $\begin{array}{l}t_{0,05} \\
\text { I.C. }(95 \%)\end{array}$ & $\begin{array}{c}4,69 \\
(0,94 ; 23,40)\end{array}$ & $\begin{array}{c}0,13 \\
(0,01 ; 1,71)\end{array}$ & $\begin{array}{c}0,26 \\
(0,05 ; 1,34)\end{array}$ & $\begin{array}{c}0,15 \\
(0,03 ; 0,70)\end{array}$ \\
\hline $\begin{array}{l}t_{0,05 B} \\
\text { I.C. }(95 \%)\end{array}$ & $\begin{array}{c}2,79 \\
(1,84 ; 13,75)\end{array}$ & $\begin{array}{l}* \\
*\end{array}$ & $\begin{array}{c}0,16 \\
(0,07 ; 0,67)\end{array}$ & $\begin{array}{c}0,07 \\
(0,05 ; 0,30)\end{array}$ \\
\hline $\begin{array}{l}t_{0,10 B} \\
\text { I.C. }(95 \%)\end{array}$ & $\begin{array}{c}9,26 \\
(2,49 ; 34,43)\end{array}$ & $\begin{array}{c}0,47 \\
(0,06 ; 3,95)\end{array}$ & $\begin{array}{c}0,66 \\
(0,17 ; 2,53)\end{array}$ & $\begin{array}{c}0,34 \\
(0,10 ; 1,20)\end{array}$ \\
\hline $\begin{array}{l}t_{0,10 B} \\
\text { I.C. }(95 \%)\end{array}$ & $\begin{array}{c}6,71 \\
(4,19 ; 23,55)\end{array}$ & $\begin{array}{c}0,08 \\
(0,09 ; 2,17)\end{array}$ & $\begin{array}{c}0,49 \\
(0,24 ; 1,51)\end{array}$ & $\begin{array}{c}0,23 \\
(0,14 ; 0,59)\end{array}$ \\
\hline
\end{tabular}

Observações: $1 . \alpha$ é o parâmetro de escala e $\delta$ é o parâmetro de forma do modelo de Weibull.

2. *: indica que a estimativa corrigida por vício E.C.V. obtida foi negativa. 
simulação, vícios com consideráveis ordens de grandeza, evidenciando que a estimação dessas quantidades na prática deve ser realizada com um cuidado muito especial para evitar erros grosseiros. Esses vícios foram maiores nos experimentos que envolveram censuras dos tipos I e II. Com relação aos mecanismos de censura, os experimentos com censura do tipo II forneceram estimativas sensivelmente melhores (em termos do valor médio e da variabilidade) que os experimentos com censura do tipo I. Já o aumento da taxa de censura foi um fator agravante para os experimentos cujos objetivos foram estimar o MTTF e os percentis, visto que uma alta taxa de censura $(50 \%)$ provocou grandes desvios nas E.M.V.s dessas quantidades.

Com relação ao uso do procedimento de reamostragem Bootstrap, este se mostrou bastante eficiente na redução do vício dos E.M.V.s do MTTF e, principalmente, dos percentis, melhorando suas estimativas tanto em valor médio como em termos da variabilidade quando comparadas às E.M.V.s.

Finalmente, a Tabela 6 resume o que pode ser de grande utilidade para o planejamento de experimentos de testes de vida, desde que o objetivo destes seja a estimação de quantidades como o MTTF e percentis. Ela fornece os tamanhos mínimos de amostra que foram utilizados para obter estimativas não viciadas para as quantidades de interesse, de acordo com os experimentos de simulação realizados. Por exemplo, para um experimento de testes de vida com taxa de censura do tipo II de $25 \%$, e parâmetro de forma $\delta$ igual a 1,00 , foi utilizada uma amostra de tamanho igual a 200 para se obter estimativas de máxima verossimilhança não viciadas do percentil $5 \%$. Por outro lado, utilizando o E.C.V. por reamostragem Bootstrap, foi necessária somente uma amostra de tamanho igual a 50 para que as estimativas fossem não viciadas.

Ao analisar os resultados desta tabela podemos observar fortes evidências de que experimentos de durabilidade podem ser realizados com o uso de poucas unidades amostrais. No entanto, eles fornecerão estimativas mais precisas de quantidades como o MTTF e os percentis, se na modelagem dos dados for realizado um estudo de redução do vício dos estimadores de máxima verossimilhança dessas quantidades. É importante ressaltar que os resultados apresentados neste texto em nenhum momento permitiram avaliar a variabilidade associada a essas estimativas, o que é de extrema relevância na prática. Portanto, apesar dos resultados indicarem a possibilidade de redução do tamanho da amostra em experimentos de
Tabela 6. Sugestão de tamanhos mínimos de amostras a serem usadas em experimentos de testes de vida para obtenção de estimativas não viciadas do MTTF e dos percentis $5 \%$ e $10 \%$.

\begin{tabular}{|c|c|c|c|c|c|c|c|}
\hline \multicolumn{2}{|c|}{$\begin{array}{c}\text { Tipo de } \\
\text { Dados }\end{array}$} & \multicolumn{2}{|c|}{$M T T F$} & \multicolumn{2}{|c|}{$5 \%$} & \multicolumn{2}{|c|}{$10 \%$} \\
\hline \multirow[t]{4}{*}{$\begin{array}{c}\text { Sem } \\
\text { Censura } \\
\end{array}$} & $\delta$ & EMV & ECV & EMV & ECV & EMV & ECV \\
\hline & 0,75 & 25 & 25 & $>200$ & 50 & 200 & 25 \\
\hline & 1,00 & 25 & 25 & 150 & 25 & 100 & 25 \\
\hline & 1,50 & 25 & 25 & 75 & 25 & 50 & 25 \\
\hline \multirow[t]{2}{*}{$\begin{array}{c}\text { Censura } \\
\text { Tipo I }\end{array}$} & $\delta$ & EMV & $\mathrm{ECV}$ & EMV & ECV & EMV & ECV \\
\hline & 0,75 & 25 & 25 & 200 & 50 & 150 & 25 \\
\hline \multirow[t]{3}{*}{$10 \%$} & 1,00 & 25 & 25 & 150 & 25 & 100 & 25 \\
\hline & 1,50 & 25 & 25 & 75 & 25 & 50 & 25 \\
\hline & 0,75 & 50 & 50 & $>200$ & 50 & 200 & 25 \\
\hline \multirow[t]{3}{*}{$25 \%$} & 1,00 & 25 & 25 & 150 & 25 & 100 & 25 \\
\hline & 1,50 & 25 & 25 & 75 & 25 & 50 & 25 \\
\hline & 0,75 & 100 & 75 & $>200$ & 50 & 200 & 50 \\
\hline \multirow[t]{2}{*}{$50 \%$} & 1,00 & 50 & 50 & 150 & 50 & 100 & 50 \\
\hline & 1,50 & 50 & 50 & 75 & 50 & 75 & 50 \\
\hline \multirow[t]{2}{*}{$\begin{array}{c}\text { Censura } \\
\text { Tipo II }\end{array}$} & $\delta$ & EMV & $\mathrm{ECV}$ & EMV & ECV & EMV & ECV \\
\hline & 0,75 & 25 & 25 & $>200$ & 50 & 200 & 50 \\
\hline \multirow[t]{3}{*}{$10 \%$} & 1,00 & 25 & 25 & 150 & 50 & 150 & 25 \\
\hline & 1,50 & 25 & 25 & 100 & 25 & 75 & 25 \\
\hline & 0,75 & 25 & 50 & $>200$ & 50 & 200 & 50 \\
\hline \multirow[t]{3}{*}{$25 \%$} & 1,00 & 25 & 25 & 200 & 50 & 150 & 25 \\
\hline & 1,50 & 25 & 25 & 100 & 50 & 75 & 25 \\
\hline & 0,75 & 50 & 150 & $>200$ & 50 & $>200$ & 50 \\
\hline \multirow[t]{2}{*}{$50 \%$} & 1,00 & 50 & 75 & $>200$ & 75 & 150 & 25 \\
\hline & 1,50 & 25 & 50 & 150 & 25 & 75 & 25 \\
\hline
\end{tabular}

durabilidade, é necessário avaliar o impacto dessa diminuição na variabilidade das estimativas de interesse. Finalmente, é importante ressaltar que apesar de o método descrito neste trabalho ter sido aplicado em experimentos com população homogênea, ele ainda pode ser utilizado em experimentos de testes de vida acelerados que são muito comuns nas aplicações industriais.

\section{Agradecimentos}

Os autores agradecem aos avaliadores anônimos pelas valiosas sugestões. 
COLOSIMO, E. A.; SILVA, A. F.; CRUZ, F. R. B. Bias evaluation in the proportional hazards model. Journal of Statistical Computation and Simulation, v. 65, p. 191-201, 2000.

COLOSIMO, E. A.; HO, L. L. Practical approach to interval estimation for the Weibull mean lifetime. Quality Engineering, v. 12, n.2, p.161-167, 1999.

COX, D. R.; SNELL, E. J. A general definition of residuals (with discussion). Journal of the Royal Statistical Society, serie B, v. 30, p.248-275, 1968.

COX, D. R.; HINKLEY, D. V. Theoretical Statistics. New York: Chapman and Hall, 1974.

EFRON, B. Bootstrap methods: another look at the jackknife. Annals of Statistics, v. 7, p. 1-26, 1979.

EFRON, B. Censored data and the Bootstrap. Journal of the American Statistical Association, v. 76, p. 312-319, 1981.

EFRON, B.; TIBSHIRANI, R. Bootstrap methods for standard errors, confidence intervals, and other measures of statistical accuracy (with discussion). Statistical Science, v. 1, p. 54-96, 1986.

EFRON, B.; TIBSHIRANI, R. An introduction to the Bootstrap. New York: Chapman and Hall, 1993.

FERRARI, S. L. P.; SILVA, A. F. Analytical and resampling-based bias corrections for one-parameter models.
Brazilian Journal of Probability and Statistical, v. 13, n. 1, p. 13-27, 1999.

JACQUELIN, J. Generalization of the method of maximum likelihood. IEEE Transactions on Dielectrics and Electrical Insulation, v. 28, n. 1, p. 65-72, 1993.

KAHLE, W. Bartlett corrections for the Weibull distribution. Metrika, v. 43, p. 257-263, 1996.

LAWLESS, J. F. Statistical models and methods for life time data. New York: Wiley \& Sons. 1982.

LEWIS, E. E. Introduction to reliability engineering. $2^{\mathrm{a}}$ ed., John Wiley \& Sons, New York, 1996.

MATLAB Release Notes. Version 6.5 Release 13. The MathWorks, Inc., 2003.

NELSON, W. Applied life data analysis. John Wiley \& Sons, New York, 1982.

NELSON, W. Accelerated testing, statistical models, test plans and data analysis. John Wiley \& Sons, New York, 1990.

NETER, J.; WASSERMAN, W.; KUTNER, M. H. Applied linear regression models. Mc Graw Hill, 1996.

RIBEIRO, J. L. Confiabilidade de sistemas. Porto Alegre: PPGEP/UFGRS, 1995.

ROSS, R. Formulas to describe the bias and standard deviation of the ML-estimated Weibull shape parameter. IEEE Transactions on Dielectrics and Electrical Insulation, v. 1, p. 247-253, 1994.

\title{
UNBIASED ESTIMATOR FOR MTTF AND QUANTILES OBTAINED FROM WEIBULL'S REGRESSION MODEL
}

\begin{abstract}
Consider a reliability model described by a Weibull regression, whose parameters are estimated by the maximum likelihood method. These parameters are used to estimate other quantities of interest such as MTTF (mean time to failure) and quantiles, which in turn play an important role in a reliability analysis.

This paper proposes improvements in MTTF and quantile estimators, whose maximum likelihood estimates (MLEs) are biased, particularly in cases involving small sample sizes. A Bootstrap procedure is presented to correct such biases. The proposed procedure is evaluated based on simulations in which the sample size, censoring mechanisms, and percentiles of censored data are varied. These simulations allow one to quantify the bias, since the analytical expression is highly complex. The proposed procedure is illustrated through an example.
\end{abstract}

Keywords: bias correction, Bootstrap, MTTF, quantiles, weibull regression model. 